LBL-34512

$\mathrm{UC}-405$

\title{
An Optimal Constrained Linear Inverse Method for Magnetic Source Imaging
}

\author{
Paul Hughett
}

\author{
Department of Electrical Engineering and Computer Sciences \\ University of California, Berkeley \\ and \\ Life Sciences Division \\ Lawrence Berkeley Laboratory \\ University of California \\ One Cyclotron Road \\ Berkeley CA 94720
}

This work was supported in part by a gift from the Advanced Technology Systems Division of the General Electric Company and in part by the Director, Office of Energy Research, Office of Health and Environmental Research, Medical Applications and Biophysical Research Division of the Department of Energy under Contract No. DE-AC0376SF00098. 
Optimal Constrained Linear Inverse Method

\section{Summary of Symbols Used}

A $=\mathbf{E} \mathbf{q q}^{T}$. The a priori covariance matrix of the source amplitudes.

$\hat{\mathbf{A}} \quad=E(\hat{\mathbf{q}}-E \hat{\mathbf{q}})(\hat{\mathbf{q}}-E \hat{\mathbf{q}})^{T}$. The a posteriori covariance matrix of the source amplitudes.

$a_{k} \quad=\mathbf{v}_{k}^{T} \mathbf{q}$. The component of $\mathbf{q}$ in the direction of the $k$ th rig." singular vector of F.

$\alpha_{i j}^{2} \quad=\mathrm{E} q_{i} q_{j}$. The a priori cross-covariance of source amplitudes $q_{i}$ and $q_{j}$. The $(i, j)$ th entry of $\mathbf{A}$.

$\hat{a}_{i j}^{2} \quad=\mathrm{E} \hat{q}_{i} \hat{q}_{j}$. The a posteriori cross-covariance of the estimated source amplitudes $\hat{q}_{i}$ and $\hat{q}_{j}$. The $(i, j)$ th entry of $\hat{\mathbf{A}}$.

$\alpha_{k}^{2} \quad=\mathrm{E} a_{k}^{2}=\mathrm{E}\left\|\mathbf{v}_{k}^{T} \mathbf{q}\right\|^{2}$. The variance of $a_{k}$.

$\vec{B}(\vec{r}) \quad$ Magnetic field as a function of position.

$\vec{B}_{n}(\vec{r}) \quad$ Magnetic field due to the $n$th source.

$b_{m} \quad$ Magnetic field measurement (including noise) at the $m$ th detector.

b The vector of field measurements $b_{m}$.

B $=\mathbf{E b b}^{T}=\mathbf{F A F}^{T}+\mathbf{F} \boldsymbol{\Gamma}+\mathbf{\Gamma}^{T} \mathbf{F}^{T}+\boldsymbol{\Sigma}$. The covariance of the measurement vector b.

$c_{k} \quad$ The $k$ th weighting coefficient in the weighted pseudoinverse.

$x^{2}=(\mathbf{b}-\mathbf{F} \hat{\mathbf{q}})^{T} \boldsymbol{\Sigma}^{-1}(\mathbf{b}-\mathbf{F} \hat{\mathbf{q}})$. The residual difference between the measured and reconstructed magnetic fields.

E Expectation of a random variable or a random vector.

e $\quad=\hat{\mathbf{q}}-\mathbf{q}$. The difference between the true and reconstructed current distributions.

$e^{2} \quad=\|\mathbf{e}\|^{2}=\|\hat{\mathbf{q}}-\mathbf{q}\|^{2}$. The reconstruction error, defined as the squared difference between the true and reconstructed current distributions.

$\eta^{2} \quad=\mathrm{E}\|\mathbf{e}\|^{2}=\mathrm{E}\|\hat{\mathbf{q}}-\mathbf{q}\|^{2}$. The mean reconstruction error.

$F_{m n} \quad=\partial b_{m} / \partial q_{n}$. Coefficient relating the field measurement $b_{m}$ to the source amplitude $q_{n}$.

F The matrix with entries $F_{m n}=\partial b_{m} / \partial q_{n}$ relating the field measurement vector $\mathbf{b}$ to the source amplitude vector $\mathbf{q}$. 
Optimal Constrained Linear Inverse Method

$\vec{r}_{m} \quad$ Position of the $m$ th detector.

$\mathbf{R} \quad \mathbf{R R}^{T}$ is the Cholesky decomposition of $\mathbf{A}$.

$\vec{s}_{m} \quad$ Unit vector in direction of the field component sampled by the $m$ th detector.

$\mathbf{S} \quad \mathbf{S S}^{T}$ is the Cholesky decomposition of $\boldsymbol{\Sigma}$.

$s_{k} \quad=\mathbf{u}_{k}^{T} \mathbf{w}$. The component of $\mathbf{w}$ in the direction of the $k$ th left singular vector of F.

$\boldsymbol{\Sigma}=\mathbf{E} \mathbf{w} \mathbf{w}^{T}$. The covariance matrix of the noise amplitude vector $\mathbf{w}$.

$\sigma_{i j}^{2} \quad=\mathrm{E} w_{i} w_{j}$. The covariance of noise amplitudes $w_{i}$ and $w_{j}$. The $(i, j)$ th entry of $\boldsymbol{\Sigma}$.

$\sigma_{k}^{2} \quad=E s_{k}^{2}$. The variance of $s_{k}$.

$\mathbf{u}_{k} \quad$ The $k$ th left singulas vector of $\mathbf{F}$.

$\mathbf{v}_{k} \quad$ The $k$ th right sigular vector of $\mathbf{F}$.

$w_{m} \quad$ Noise amplitude in the $m$ th detector. 


\section{Optimal Constrained Linear Inverse Method}

If the statistics of the measurement noise are available, maximum-likelihood (ML) methods are appropriate [15]. These methods maximize the likelihood of obtaining the measured fields given the reconstructed current distribution; if the noise is jointly Gaussian, they also minimize the squared difference between the measured and reconstructed fields, weighted to reflect the a priori noise variance.

If prior anatomical information is available from transmission tomography or magnetic resonance imaging, then it may be possible to define the a priori source variance as a function of position. Then minimum mean-square error (MMSE) methods are appropriate; they minimize the mean (average) squared difference between the true and reconstructed current distributions. Shim and Cho [16] have developed methods using a weighted pseudoinverse but their methods are optimal and useful only when the a priori source variance is constant. Helstrom [17] has developed and applied an MMSE method for inage restoration; Smith et al. [18] have developed and tested an MMSE method for magnetic source imaging with general a priori source variances.

The present paper presents a unified development of the MNLS, ML, weighted pseudoinverse, and MMSE methods and shows that the weighted pseudoinverse, maximum likelihood, and MNLS methods can all be obtained as special cases of the MMSE method by an appropriate choice of priors. Formulas for the mean reconstruction error, mean residual, and a posteriori variance (or confidence limits) are derived. The paper also discusses efficient computer algorithms for the MMSE method and presents simulation results.

Oh et al. [19] have taken a different approach to the use of prior information by using the method of alternating projections to obtain "line-like" or filamentary reconstructions consistent with the measurements.

Most of the examples in this paper are taken from magnetoencephalography, but the methods should apply to magnetocardiography and geophysics as well. Furthermore, the methods should generalize to other reconstruction problems including positron emission tomography (PET) and single photon emission computed tomography (SPECT).

\section{An Approach to the Problem}

The choice of the mathematical model used to represent the prior knowledge is a compromise between several desiderata: First, the model should iead to an analytical or mumerical solution method for the inverse problem; second, the parameters of the model should be physically meaningful, so that the user has some intuition of their meaning; third, the required parameters should be directly measureable (at least in principle) by some calibration experiment; fourth, the method should involve an objective criterion for correctness or optimality; and fifth, the solution method should admit some statistical test of goodness of fit between the model found and the measurements made. This paper considers one particular model that satisfies these requirements.

It is useful to exploit the fact that the magnetic field is a linear function of the current distribution. That is, the field due to a weighted sum of current sources is equal to the same weighted sum of the fields due to the individual sources. Thus, it is convenient to represent 
Optimal Constrained Linear Inverse Method

elementary sources $\vec{J}_{n}(\vec{r})$ to obtain

$$
\vec{J}(\vec{r})=\sum_{n=1}^{N} q_{n} \vec{J}_{n}(\vec{r})
$$

Each elementary source $\vec{J}_{n}(\vec{r})$ is a vector-valued function giving the vector current density at any position $\vec{r}$. Note that the sources are at positions fixed a priori; their positions are not free parameters to be estimated. Only the source amplitudes are unknown and must be estimated.

There are many possibilities for the set of elementary sources $\vec{J}_{n}(\vec{r})$, depending on the assumptions made about the unknown distribution $\vec{J}(\vec{r})$.

If the unknown distribution is assumed to be well-described by a few localized sources, then it is reasonable to use a few current dipoles whose positions, magnitudes, and orientations are to be determined. The magnetic field measurements are nonlinear functions of the source positions and iterative solution is generally necessary. The methods described in this paper assume positions fixed a priori and are not directly useful; they could possibly be used to find optimal source amplitudes at each step of an iterative scheme for improving the source position estimates. If, however, the source locations are known a priori and only the amplitudes are unknown, then the methods of this paper would be applicable.

If the unknown distribution is assumed to be smooth, then it is reasonable to expand it in a set of basis functions; these basis functions are the elementary current sources. A grid of current dipoles or a finite element mesh [20, 21] define localized elementary sources; lead fields [12], multipole expansions [22], or Fourier basis functions define nonlocalized elementary sources. Provided that the sources have fixed positions, the magnetic field measurements are linear functions of the source amplitudes and the linear methods developed in this paper can be used to solve the inverse problem. Any of the above basis functions could be used, though this paper considers only current dipoles in detail.

The vector $q$ of source amplitudes $q_{n}$ is assumed to be a random vector with mean zero and covariance matrix $\mathrm{A}=\mathrm{E} \mathrm{qq}^{T}$ with entries $\alpha_{i j}^{2}=\mathrm{E} q_{i} q_{j}$. If the expected amplitudes are not zero-mean, the shifted vector $\tilde{q}=q-E q$ is zero-mean and can be used instead. Since $\mathbf{A}$ is a covariance matrix, it is symmetric and positive semidefinite.

Suppose that $\rho(\vec{r})$ is the resistivity as a function of position $\vec{r}$. If we normalize each source such that

$$
\int_{R^{3}} \rho(\vec{r})\left|\vec{J}_{n}(\vec{r})\right|^{2} d^{3} \vec{r}=1
$$

then the expected (or average) power dissipation of the $n$th source $q_{n} \vec{J}_{n}$ is

$$
\begin{aligned}
P_{n} & =E \int_{R^{3}} \rho(\vec{r})\left|q_{n} \vec{J}_{n}(\vec{r})\right|^{2} d^{3} \vec{r} \\
& =\left(E q_{n}^{2}\right) \int_{R^{3}} \rho(\vec{r})\left|\vec{J}_{n}(\vec{r})\right|^{2} d^{3} \vec{r} \\
& =\alpha_{n n}^{2} .
\end{aligned}
$$


noise power of the $m$ th detector. Since $\boldsymbol{\Sigma}$ is a covariance matrix, it is symmetric and positive semidefinite. If the noise is uncorrelated between sensors, then $\boldsymbol{\Sigma}$ is diagonal.

The cross-covariance between the source and noise amplitude vectors is $\Gamma=E \mathrm{qw}^{T}$ with entries $\gamma_{n m}=E q_{n} w_{m}$. In many applications, there will be no source-noise correlation and $\Gamma$ will be zero.

Given these definitions, the measurement covariance is

$$
\mathbf{B}=\mathbf{E} \mathbf{b b}^{T}=\mathbf{F A F} \mathbf{F}^{T}+\mathbf{F} \boldsymbol{\Gamma}+\Gamma^{T} \mathbf{F}^{T}+\mathbf{\Sigma}
$$

In the case that $\boldsymbol{\Gamma}=0$, this simplifies to $\mathbf{B}=\mathbf{F A F} \mathbf{F}^{T}+\boldsymbol{\Sigma}$ in which $\mathbf{F A} \mathbf{F}^{T}$ is the variance (or power) due to the sources and $\boldsymbol{\Sigma}$ is the variance due to the noise. Thus we may define the signal-to-noise ratio as

$$
S N R=\frac{\operatorname{Tr}\left(\mathbf{F A} \mathbf{F}^{T}\right)}{\operatorname{Tr}(\boldsymbol{\Sigma})}
$$

Note that the only assumptions made about the elementary sources $\vec{J}_{n}$ are that the sources are fixed in position a priori and that the superposition principle of electromagnetics holds.

Dipole Sources: In the particular case that each source $\vec{J}_{n}$ is a current dipole with moment $\vec{q}_{n}$ at position $\vec{p}_{n}$ (and ignoring the volume currents), the field is

$$
\vec{B}_{n}(\vec{r})=\frac{\mu_{0}}{4 \pi} \cdot \frac{\vec{q}_{n} \times\left(\vec{r}-\vec{p}_{n}\right)}{\left\|\vec{r}-\vec{p}_{n}\right\|^{3}}
$$

and the response matrix has entries

$$
F_{m n}=\frac{\mu_{0}}{4 \pi} \cdot \frac{\vec{s}_{m} \cdot \vec{q}_{n} \times\left(\vec{r}_{m}-\vec{p}_{n}\right)}{\left\|\vec{r}_{m}-\vec{p}_{n}\right\|^{3}} .
$$

A "rotating" dipole of unknown orientation at a given position may be represented as two or three orthogonal fixed dipoles. The fixed dipoles may be oriented along the coordinate axes; normal and tangential to the cortical surface; or along the principal axes of the assumed probability density for the rotating dipole. In the last case, the fixed clipoles will be uncorrelated.

In magnetoencephalography, a dipole source will ordinarily be oriented normal to the cortical surface and the direction of of $\vec{J}_{n}$ may be chosen to match. If the surface is strongly curved, a normal dipole with large expected amplitude and two tangential dipoles with smaller expected amplitude may be used.

Assumption A: We pause here to introduce a technical assumption to be called assumption A - that will useful later. Precisely stated, we will assume hereafter that that 
Conversely, suppose that a map of expected power density can be derived from phys. iological principles. Then dividing this map into small regions of approximately constant power density and assigning a singleton, pair, or triplet of orthogonal dipoles to each region yields a set of dipoles to model the unknown source distribution. The orientation of the dipole triplet should be chosen to match the principal axes of the most plausible a priori distribution; if the distribution is spherical, then the orientation does not matter and may be chosen for convenience (e.g. to match the coordinate axes). The integrated power density in each region defines the total expected power or a priori variance for the corresponding triplet (or pair or singletor ); if physiology does not suggest a reasonable subdivision over the members of the tripler, eyual variances seem as plausible as anything else. Then the source variances taken together define the diagonal of the a priori source covariance matrix $\mathbf{A}$.

For example, sources located in the grey matter (as determined by magnetic resonance imaging) might be oriented normal to the cortex and assigned twice the expected power of sources in the white matter or cerebrospinal fluid; sources outside the head would be omitted entirely.

Uniform Priors: In the case of "uniform priors," the approximate amplitude of the elementary sources is known but there is no basis for believing that any one is more active than any other; and the same is true of the noise amplitudes. That is, the source amplitudes are independent and identically distributed; so are the noise amplitudes. Then every source has the same expected activity $\alpha^{2}$, the covariance matrix $\mathbf{A}$ takes the form $\alpha^{2} \mathbf{I}$, and the map of expected activity is uniform; the noise covariance $\boldsymbol{\Sigma}$ takes the form $\sigma^{2} I$ and the cross-covariance $\Gamma$ is zero.

\section{The Inverse Problem}

The inverse problem is to find a "best" estimate $\hat{q}$ of the unknown source amplitude vector $q$ from given values for the response matrix $\mathbf{F}$, the field measurements $\mathbf{b}$, and perhaps other information such as the noise covariance $\boldsymbol{\Sigma}$ and the source covariance $\mathbf{A}$.

This paper considers only linear inverse methods. That is, the best estimate $\hat{\mathbf{q}}$ is always computed in the form

$$
\hat{\mathbf{q}}=\mathbf{H b}
$$

where $\mathbf{H}$ is a linear operator possibly depending on $\mathbf{A}, \boldsymbol{\Sigma}, \boldsymbol{\Gamma}$, or other prior information.

The simple solution $\hat{\mathbf{q}}=\mathbf{F}^{-1} \mathbf{b}$ does not work in general for inverse problems; $\mathbf{F}$ is rarely invertible and usually rectangular. The inverse problem is of ten both overdetermined in the sense that no solution $\hat{q}$ exactly solves $\mathbf{F q}=\mathbf{b}$ and underdetermined in that many different values of $\mathbf{q}$ provide equally close approximations. Different criteria for the "best" approximation lead to different inverse methods.

The least squares (LS) criterion [26] is to minimize the residual

$$
\varepsilon^{2}=\|\mathbf{b}-\mathbf{F} \hat{\mathbf{q}}\|^{2},
$$


be computed for any specific $\mathbf{b}$; only its mean value $\eta^{2}$ can be determined. The mean square error $\eta^{2}$ depends on the source and noise statistics, ;o both of these must be known (or assumed) a priori.

For a given filter $\mathbf{H}$, the mean square error takes the form

$$
\begin{aligned}
\eta^{2} & =\mathbf{E}\|\mathbf{H}(\mathbf{F q}+\mathbf{w})-\mathbf{q}\|^{2} \\
& =\mathbf{E}\|(\mathbf{H F}-\mathbf{I}) \mathbf{q}\|^{2}+\mathbf{E}\|\mathbf{H} \mathbf{w}\|^{2}+2 \mathbf{E}(\mathbf{H w})^{T}(\mathbf{H F}-\mathbf{I}) \mathbf{q} \\
& =\operatorname{Tr}\left((\mathbf{H F}-\mathbf{I}) \mathbf{A}(\mathbf{H F}-\mathbf{I})^{T}+\mathbf{H} \mathbf{\Sigma} \mathbf{H}^{T}+2(\mathbf{H F}-\mathbf{I}) \Gamma \mathbf{H}^{T}\right) .
\end{aligned}
$$

Also of interest is the function

$$
\rho^{2}=\hat{\mathbf{q}}^{T} \mathbf{A}^{-1} \hat{\mathbf{q}},
$$

which is a measure of the goodness of fit between the reconstructed current distribution and the a priori current distribution. Since $\rho^{2}$ increases as the reconstruction becomes less likely relative to the priors, it will be called the "surprise." For given $\mathbf{b}$ and $\mathbf{H}$, it has the value

$$
\begin{aligned}
\rho^{2} & =\hat{\mathbf{q}}^{T} \mathbf{A}^{-1} \hat{\mathbf{q}} \\
& =\left(\mathbf{b}^{T} \mathbf{H}^{T} \mathbf{R}^{-T}\right)\left(\mathbf{R}^{-1} \mathbf{H} \mathbf{b}\right) \\
& =\left\|\mathbf{R}^{-1} \mathbf{H} \mathbf{b}\right\|^{2} .
\end{aligned}
$$

The mean surprise indicates to what extent the reconstruction filter uses the prior information rather than the given measurements; it has the value

$$
\begin{aligned}
E \rho^{2} & =E\left\|\mathbf{R}^{-1} \mathbf{H b}\right\|^{2} \\
& =\operatorname{Tr}\left(\mathbf{R}^{-1} \mathbf{H B H} \mathbf{H}^{T} \mathbf{R}^{-T}\right) \\
& =\operatorname{Tr}\left(\left(\mathbf{R}^{-1} \mathbf{H L}\right)\left(\mathbf{R}^{-1} \mathbf{H L}\right)^{T}\right) \\
& =\left\|\left(\mathbf{R}^{-1} \mathbf{H L}\right)\right\|_{F}^{2} .
\end{aligned}
$$

\section{Minimum-Norm Least Squares Method}

The Moore-Penrose generalized inverse (sometimes known simply as the pseudoinverse) is a generalization of the matrix inverse to arbitrary rectangular matrices. It was first reported by Moore [28] in 1920 as the unique matrix satisfying certain algebraic conclitions not relevant here, and rediscovered by Penrose [29] in 1955 using different but ecquivalent algebraic conditions. Of importance to the present paper is the fact [30] that it computes the MNLS solution.

The Moore-Penrose inverse can be easily computed from the singular value decomposition (SVD) $[24,25]$ of the response matrix $\mathbf{F}$. To fix notation, the SVD theorem states that the $M \times N$ response matrix $\mathbf{F}$ can be decomposed in the form

$$
\mathbf{F}=\mathbf{U} \boldsymbol{\Lambda} \mathbf{V}^{T}=\sum_{k=1}^{k} \lambda_{k} \mathbf{u}_{k} \mathbf{v}_{k}^{T}
$$


those errors without introducing any new ones, and generalizes their result by allowing correlations between noise and source amplitudes $(\Gamma \neq 0)$.

Shim and Cho have applied the optimal truncated pseudoinverse method (OTPIM) and optimal weighted pseudoinverse method (OWPIM) to PET reconstruction; Jeffs, Leahy, and Singh [11] have used the optimal truncated pseudoinverse for magnetic source imaging of the brain.

To determine the optimal $c_{k}$, write the source and noise vectors in terms of the singular vectors of $\mathbf{F}$ to obtain

$$
\mathbf{q}=\sum_{k=1}^{N} a_{k} \mathbf{v}_{k} \quad \text { and } \quad \mathbf{w}=\sum_{k=1}^{M} s_{k} \mathbf{u}_{k} .
$$

Note that $a_{k}=\mathbf{v}_{k}^{T} \mathbf{q}$ and $s_{k}=\mathbf{u}_{k}^{T} \mathbf{w}$, so that $E a_{k}=0$ and $E s_{k}=0$. Define the covariances

$$
\begin{aligned}
\alpha_{k}^{2} & =\mathrm{E} a_{k}^{2}=\mathbf{v}_{k}^{T} \mathbf{A} \mathbf{v}_{k}, & & k=1, \ldots, N \quad ; \\
\sigma_{k}^{2} & =\mathrm{E} s_{k}^{2}=\mathbf{u}_{k}^{T} \mathbf{\Sigma} \mathbf{u}_{k}, & & k=1, \ldots, M \quad ; \text { and } \\
\gamma_{k} & =\mathrm{E} a_{k} s_{k}=\mathbf{v}_{k}^{T} \Gamma \mathbf{u}_{k}, & & k=1, \ldots, K^{-1}
\end{aligned}
$$

which are the diagonal entries of the rotated covariance matrices $\mathbf{V}^{T} \mathbf{A V}, \mathbf{U}^{T} \mathbf{\Sigma} \mathbf{U}$, and $\mathrm{V}^{T} \Gamma \mathrm{U}$ respectively. The notation here may be a bit confusing; the symbols $\alpha_{i j}^{2}, \sigma_{i j}^{2}$, and $\gamma_{i j}$ with two subscripts denote the entries of the unrotated covariance matrices $\mathbf{A}, \boldsymbol{\Sigma}$, and $\Gamma$.

The optimal estimate of $\mathbf{q}$ is $\hat{\mathbf{q}}=\mathbf{F}^{w} \mathbf{b}$ with error

$$
\begin{aligned}
\mathbf{e} & =\hat{\mathbf{q}}-\mathbf{q}=\mathbf{F}^{w} \mathbf{b}-\mathbf{q}=\mathbf{F}^{w}(\mathbf{F q}+\mathbf{w})-\mathbf{q} \\
& =\mathbf{F}^{w} \mathbf{F} \mathbf{q}-\mathbf{q}+\mathbf{F}^{w} \mathbf{w} \\
& =\mathbf{F}^{w} \mathbf{F} \sum_{k=1}^{N} a_{k} \mathbf{v}_{k}-\sum_{k=1}^{N} a_{k} \mathbf{v}_{k}+\mathbf{F}^{w} \sum_{k=1}^{M} s_{k} \mathbf{u}_{k} ;
\end{aligned}
$$

and since $\mathbf{v}_{K+1}, \ldots, \mathbf{v}_{N}$ are in the null space of $\mathbf{F}$ and $\mathbf{u}_{K+1}, \ldots, \mathbf{u}_{M}$ are in the mull space of $\mathbf{F}^{\left({ }^{\prime \prime}\right.}$,

$$
\begin{aligned}
\mathbf{e} & =\mathbf{F}^{w} \mathbf{F} \sum_{k=1}^{K} a_{k} \mathbf{v}_{k}-\sum_{k=1}^{N} a_{k} \mathbf{v}_{k}+\mathbf{F}^{w} \sum_{k=1}^{K} s_{k} \mathbf{u}_{k} . \\
& =\sum_{k=1}^{K}\left[\left(c_{k}-1\right) a_{k}+\frac{c_{k} s_{k}}{\lambda_{k}}\right] \mathbf{v}_{k}-\sum_{k=K+1}^{N} a_{k} \mathbf{v}_{k} .
\end{aligned}
$$

Then the mean squase error is

$$
\eta^{2}=\mathbf{E e}^{T} \mathbf{e}=\sum_{k=1}^{K}\left[\left(c_{k}-1\right)^{2} \alpha_{k}^{2}+2 c_{k}\left(c_{k}-1\right) \gamma_{k} / \lambda_{k}+c_{k}^{2} \sigma_{k}^{2} / \lambda_{k}^{2}\right]+\sum_{k=K+1}^{N} \alpha_{k}^{2} .
$$


Optimal Constrained Linear Inverse Method

The mean surprise is

$$
\begin{aligned}
E \rho^{2} & =\left\|\mathbf{R}^{-1} \mathbf{H L}\right\|_{F}^{2} \\
& =\left\|\mathbf{R}^{-1}\left(\sum_{k=1}^{K} c_{k} \lambda_{k}^{-1} \mathbf{v}_{k} \cdot \mathbf{u}_{k}^{T}\right) \mathbf{L}\right\|_{F}^{2} \\
& =\left\|\sum_{k=1}^{K} c_{k} \lambda_{k}^{-1}\left(\mathbf{R}^{-1} \mathbf{v}_{k}\right)\left(\mathbf{L}^{T} \mathbf{u}_{k}\right)^{T}\right\|_{F}^{2} .
\end{aligned}
$$

\section{Optimal Constrained Linear Inverse Method}

The Shim-Cho weighted pseudoinverse is optimal (in the MMSE sense) over all possible weighted pseudoinverses but is not, in general, optimal over all possible linear estimators of the form $\hat{\mathbf{q}}=\mathbf{H b}$.

The optimal constrained linear estimator is given by the matrix $\mathbf{H}$ that minimizes

$$
\begin{aligned}
\eta^{2} & =E\|\hat{\mathbf{q}}-\mathbf{q}\|^{2}=E\|\mathbf{H} \mathbf{b}-\mathbf{q}\|^{2} \\
& =E\|\mathbf{H}(\mathbf{F q}+\mathbf{w})-\mathbf{q}\|^{2} \\
& =E\|(\mathbf{H F}-\mathbf{I}) \mathbf{q}+\mathbf{H} \mathbf{w}\|^{2}
\end{aligned}
$$

Now consider a variation $\mathbf{H}+\epsilon \delta \mathbf{H}$ where $\epsilon$ is a scalar and $\delta \mathbf{H}$ is a matrix to obtain

$$
\begin{aligned}
\eta^{2}= & E\|(\mathbf{H F}+\epsilon \delta \mathbf{H F}-\mathbf{I}) \mathbf{q}+\mathbf{H} \mathbf{w}+\epsilon \delta \mathbf{H} \mathbf{w}\|^{2} \\
= & E\|(\mathbf{H F}-\mathbf{I}) \mathbf{q}+\mathbf{H} \mathbf{w}+\epsilon \mathbf{H}(\mathbf{F q}+\mathbf{w})\|^{2} \\
= & \mathrm{E}\|(\mathbf{H F}-\mathbf{I}) \mathbf{q}+\mathbf{H} \mathbf{w}\|^{2} \\
& +2 \epsilon \mathrm{E}(\mathbf{F q}+\mathbf{w})^{T} \delta \mathbf{H}^{T}[(\mathbf{H F}-\mathbf{I}) \mathbf{q}+\mathbf{H} \mathbf{w}] \\
& +\epsilon^{2} \mathrm{E}\|\delta \mathbf{H}(\mathbf{F q}+\mathbf{w})\|^{2} \\
= & c_{0}+c_{1} \epsilon+c_{2} \epsilon^{2}
\end{aligned}
$$

for scalars $c_{0}, c_{1}$, and $c_{2}$ which depend on $\delta \mathbf{H}$.

Now $\mathbf{H}$ can minimize $\eta^{2}$ only if $c_{1}$ is zero for any value of $\delta \mathbf{H}$. To see this, assume on the contrary that there is some non-zero $\delta \mathbf{H}$ such that $c_{1}$ is not zero. Assumption A ensures that the constant $c_{2}=E\|\delta \mathbf{H}(\mathbf{F q}+\mathbf{w})\|^{2}$ is positive whenever $\delta \mathbf{H}$ is not zero. Then $\eta^{2}$ has a unique minimum at $\epsilon_{\min }=-c_{1} /\left(2 c_{2}\right)$. But this means that $\eta^{2}$ is smaller for $\mathbf{H}+\epsilon_{\min } \delta \mathbf{H}$ than for $\mathbf{H}$. Thus, contrary to our assumption, $\mathbf{H}$ is not the minimum. Therefore, $c_{1}$ must be zero for every $\delta H$.

The positivity of $c_{2}$ also guarentees that $\eta^{2}$ has a minimum rather than a maximum. Furthermore, since $\eta^{2}$ is quadratic in $\epsilon$, the minimum is mique and there is no maximum. That is, the optimal $\mathbf{H}$ exists and is unique. 
The factor $\mathbf{\Sigma}^{-1}$ conrects for noise varinnce in the projertions, $\mathbf{F}^{T}$ backprojects, and $\left(\mathbf{A}^{-1}+\right.$ $\left.\mathbf{F}^{7} \mathbf{\Sigma}^{\cdots 1} \mathbf{F}\right)^{-1}$ filters the backprojected image.

Using the fact that

$$
\begin{aligned}
\mathbf{H F}-\mathbf{I} & =\left(\mathbf{A}^{-1}+\mathbf{F}^{T} \mathbf{\Sigma}^{-1} \mathbf{F}\right)^{-1} \mathbf{F}^{T} \mathbf{\Sigma}^{-1} \mathbf{F}-\mathbf{I} \\
& =-\left(\mathbf{A}^{-1}+\mathbf{F}^{T} \boldsymbol{\Sigma}^{-1} \mathbf{F}\right)^{-1} \mathbf{A}^{-1}
\end{aligned}
$$

and substituting into (20) yields the meas square error

$$
\begin{aligned}
\eta^{2}= & \operatorname{Tr}\left(\left(\mathbf{A}^{-1}+\mathbf{F}^{T} \mathbf{\Sigma}^{-1} \mathbf{F}\right)^{-1} \mathbf{A}^{-1} \mathbf{A} \mathbf{A}^{-1}\left(\mathbf{A}^{-1}+\mathbf{F}^{T} \mathbf{\Sigma}^{-1} \mathbf{F}\right)^{-1}\right. \\
& \left.\quad+\left(\mathbf{A}^{-1}+\mathbf{F}^{l} \mathbf{\Sigma}^{-1} \mathbf{F}\right)^{-1} \mathbf{F}^{T} \mathbf{\Sigma}^{-1} \mathbf{\Sigma} \mathbf{\Sigma}^{-1} \mathbf{F}\left(\mathbf{A}^{-1}+\mathbf{F}^{T} \mathbf{\Sigma}^{-1} \mathbf{F}\right)^{-1}\right) \\
= & \operatorname{Tr}\left(\left(\mathbf{A}^{-1}+\mathbf{F}^{l} \mathbf{\Sigma}^{-1} \mathbf{F}\right)^{-1}\right) \\
= & \operatorname{Tr}\left(\mathbf{A}-\mathbf{A} \mathbf{F}^{T}\left(\mathbf{F A} \mathbf{F}^{T}+\mathbf{\Sigma}\right)^{-1} \mathbf{F A}\right) \\
= & \operatorname{Tr}\left(\mathbf{A}-\left(\mathbf{L}^{-1} \mathbf{F A}\right)^{T}\left(\mathbf{L}^{-1} \mathbf{F A}\right)\right) \\
= & \operatorname{Tr}(\mathbf{A})-\left\|\mathbf{L}^{-1} \mathbf{F A}\right\|_{r}^{2},
\end{aligned}
$$

Alternatively, $\eta^{2}$ can be computed fror:d the a posteriori variance $\hat{\mathbf{A}}$ discusser later.

To find the mean resirlual and mean surprise, first observe that

$$
\begin{aligned}
\mathbf{H} & =\mathbf{A} \mathbf{F}^{\prime}\left(\mathbf{F A F}^{\prime}+\mathbf{\Sigma}\right)^{-1} \\
& =\mathbf{R R}^{\top} \mathbf{F}^{\prime} \mathbf{L}^{-} \mathbf{L}^{-1}
\end{aligned}
$$

and

$$
\begin{aligned}
\mathbf{I}-\mathbf{F H} & =\mathbf{I}-\mathbf{F A F}^{l}\left(\mathbf{F A F} \mathbf{F}^{T}+\mathbf{\Sigma}\right)^{-1} \\
& =\mathbf{\Sigma}\left(\mathbf{F A F}^{T}+\mathbf{\Sigma}\right)^{-1} \\
& =\mathbf{S S}^{T} \mathbf{L}^{-T} \mathbf{L}^{-1}
\end{aligned}
$$

It then follows from (18) and (23) that

$$
E_{l^{2}}^{2}=\left\|\mathbf{S}^{T} \mathbf{L}^{-T}\right\|_{F}^{2}=\left\|\mathbf{L}^{-1} \mathbf{S}\right\|_{F}^{2}
$$

and

$$
E \rho^{2}=\left\|\mathbf{R}^{\prime} \mathbf{F}^{\prime} \mathbf{L}^{\prime}\right\|_{F}^{2}=\left\|\mathbf{L}^{-1} \mathbf{F} \mathbf{R}\right\|_{F}^{2}
$$

Coaxial priors: Suppose that the covariance matrices $\mathbf{A}, \mathbf{\Sigma}$, and $\boldsymbol{\Gamma}$ all becone diagonal when they are rotated into the $\mathbf{U}$ and $\mathbf{V}$ coordinates defined by the singular vector's of $\mathbf{F}$. That is, suppose that the rotated matrices $\mathbf{V}^{\prime} \mathbf{A V}, \mathbf{U}^{T} \mathbf{\Sigma U}$, and $\mathbf{V}^{T} \mathbf{\Gamma U}$ are all diagonal. Then the matrices $\mathbf{F}, \mathbf{A}, \mathbf{\Sigma}$, and $\boldsymbol{\Gamma}$ can all be written in terms of the singulas. vectors $u_{k}$ and $v_{k}$ defined by the singular value decomposition of $\mathbf{F}$. That is,

$$
\mathbf{F}=\sum_{k=1}^{k} \lambda_{k} \cdot \mathbf{u}_{k} \mathbf{v}_{k}^{T},
$$


is useful for noise tolorant reconstruction given only rough estimates of sounce and noise anplitude

This form can be regarded as a Marquardt [34, 35] or Tikhonov [36] regularization of the pseudoinverse $\mathbf{F}^{\dagger}=\mathbf{F}^{\gamma}\left(\mathbf{F F}^{T}\right)^{-1}$. One important difference is that the value of the regularization prameter is determined by the given value of $\sigma^{2} / \alpha^{2}$ and need not be determined by experiment.

No Prior Information: The case of "no priors" may be approached by letting a" go to infinity. In this case, the OCLIM filter goes to the limit

$$
\mathbf{H}=\sum_{k=1}^{k} \lambda_{k}^{-1} \mathbf{v}_{k} \mathbf{u}_{k}^{T},
$$

which is just the Moore-Penrose inverse or the MNLS estimator. Taking the linnit as the noise goes to zero $\left(\sigma^{2} \rightarrow 0\right)$ yields the same result.

\section{A Posteriori Variance and Confidence Limits}

Given the additional assmuption that $q$ and $w$ are jointly Gaussian, it is possible to determine the a posteriori variance and hence confidence limits on the estimate $\hat{\mathbf{q}}$.

Papoulis gives the following result, which has been restated in matrix form [27]: Suppose that $\mathbf{x}$ and $\mathbf{y}$ are zero-mean, jointly Gaussian random vectors with covariance matrices $\boldsymbol{\Sigma}_{\mathbf{x x}}$ and $\boldsymbol{\Sigma}_{\mathbf{y y}}$ and cross-covariance $\boldsymbol{\Sigma}_{\mathbf{x y}}=\boldsymbol{\Sigma}_{\mathbf{y x}}^{T}$. Then, for given $\mathbf{y}, \mathbf{x}$ is normally distributed with mean

$$
\mu_{\mathbf{x}} \mid \mathbf{y}=\mathbf{\Sigma}_{\mathbf{x y}} \Sigma_{\mathbf{y} y}^{-1} \mathbf{y}
$$

and vasiance

$$
\Sigma_{x \mid y}=\Sigma_{x x}-\Sigma_{x y} \Sigma_{y y}^{-1} \Sigma_{y x}
$$

In the present problem, $\mathbf{b}$ is given and $\mathbf{q}$ is sought. Thus, we evaluate

$$
\begin{aligned}
& \boldsymbol{\Sigma}_{\mathbf{q q}}=\mathbf{E q q ^ { T }}=\mathbf{A} ; \\
& \boldsymbol{\Sigma}_{\mathbf{q} \mathbf{b}}=\mathbf{E q}(\mathbf{F q}+\mathbf{w})^{T}=\mathbf{A} \mathbf{F}^{T}+\Gamma ; \text { and } \\
& \boldsymbol{\Sigma}_{\mathbf{b b}}=\mathbf{E}(\mathbf{F q}+\mathbf{w})(\mathbf{F q}+\mathbf{w})^{T}=\mathbf{F A} \mathbf{F}^{T}+\mathbf{F} \boldsymbol{\Gamma}+\Gamma^{T} \mathbf{F}^{T}+\boldsymbol{\Sigma} .
\end{aligned}
$$

Then the mean of $q$ given $b$ is

$$
\begin{aligned}
\hat{\mathbf{q}}=\mu_{\mathbf{q} \mid \mathbf{b}} & =\boldsymbol{\Sigma}_{\mathbf{q} \mathbf{b}} \boldsymbol{\Sigma}_{\mathbf{b} \mathbf{b}}^{-1} \mathbf{b} \\
& =\left(\mathbf{A} \mathbf{F}^{T}+\Gamma\right)\left(\mathbf{F A} \mathbf{F}^{T}+\mathbf{F} \boldsymbol{\Gamma}+\Gamma^{\prime} \mathbf{F}^{T}+\mathbf{\Sigma}\right)^{-1} \mathbf{b},
\end{aligned}
$$

which is exactly the MMSE estimate derived above for $q$ and $w$ with arbitrary distributions. Furthermore, the variance of $q$ given $b$ is

$$
\begin{aligned}
\hat{\mathbf{A}} & =\boldsymbol{\Sigma}_{\mathbf{q} \mid \mathbf{b}} \\
& =\boldsymbol{\Sigma}_{\mathbf{q q}}-\boldsymbol{\Sigma}_{\mathbf{q} \mathbf{b}} \boldsymbol{\Sigma}_{\mathbf{b} \mathbf{b}}^{-1} \boldsymbol{\Sigma}_{\mathbf{b q}} \\
& =\mathbf{A}-\left(\mathbf{A} \mathbf{F}^{T}+\mathbf{\Gamma}\right)\left(\mathbf{F A} \mathbf{F}^{T}+\mathbf{F} \boldsymbol{\Gamma}+\mathbf{\Gamma}^{T} \mathbf{F}^{T}+\mathbf{\Sigma}\right)^{-1}\left(\mathbf{A} \mathbf{F}^{T}+\boldsymbol{\Gamma}\right)^{T} .
\end{aligned}
$$


1.1 Form the matrix $\mathbf{B}=\mathbf{\Sigma}+\sum_{n} \alpha_{n n}^{2} \mathbf{f}_{n} \mathbf{f}_{n}^{T}$ using $O\left(M^{2} N\right)$ flops.

1.2 Compute the Cholesky decomposition $\mathbf{L} \mathbf{L}^{T}$ of $\mathbf{B}$ in $O\left(M^{3}\right)$ flops.

2.1 Solve the system $\mathbf{L} \mathbf{L}^{T} \mathbf{p}=\mathbf{b}$ using the Cholesky decomposition in $O\left(M^{2}\right)$ flops.

2.2 Compute the solution $\hat{\mathbf{q}}=\mathbf{A F}^{T} \mathbf{p}$ in $O(M N)$ flops.

Steps 1.1 and 1.2 do not involve $\mathbf{b}$ and need be done only once for any specified configuration; steps 2.1 and 2.2 must be done for each measurement vector $\mathbf{b}$. Assuming that $M$ and $N$ are of the same order, setup (steps 1.1 and 1.2) costs $O\left(N^{3}\right)$ flops and each estimated source vector costs $O\left(N^{2}\right)$ flops.

It is also useful to compute at least the diagonal of the a posteriori source covariance

$$
\begin{aligned}
\hat{\mathbf{A}} & =\mathbf{A}-\mathbf{A F}^{T}\left(\mathbf{F A} \mathbf{F}^{T}+\mathbf{\Sigma}\right)^{-1} \mathbf{F A} \\
& =\mathbf{A}-\mathbf{A F}^{T} \mathbf{L}^{-T} \mathbf{L}^{-1} \mathbf{F A} \\
& =\mathbf{A}-\left(\mathbf{L}^{-1} \mathbf{F A}\right)^{T}\left(\mathbf{L}^{-1} \mathbf{F A}\right)
\end{aligned}
$$

Let $\mathbf{X}=\mathbf{L}^{-1} \mathbf{F A}$. Then $\mathbf{L X}=\mathbf{F A}$, which may be solved by forward substitution, and $\hat{\mathbf{A}}=\mathbf{A}-\mathbf{X}^{T} \mathbf{X}$, which may be computed directly. Note that since $\mathbf{A}$ is diagonal, computing FA simply requires multiplying each column of $\mathbf{F}$ by the appropriate diagonal entry of $\mathbf{A}$. Thus, a suitable procedure is as follows:

1.3 Form the product FA in $O(M N)$ flops.

1.4 Solve $\mathbf{L X}=$ FA by multiplication and forward substitution in $O\left(M^{3}\right)$ flops.

1.5 Compute $\hat{\mathbf{A}}=\mathbf{A}-\mathbf{X}^{T} \mathbf{X}$ directly in $O\left(N^{3}\right)$ flops.

All of these steps are independent of $\mathbf{b}$ and can be done as part of the setup; the total time required is $O\left(N^{3}\right)$ flops.

\section{Simulation Results and Discussion}

The computer implementation just described was used in a Monte Carlo simulation to compare the four inverse methods. All sources were modelled as current dipoles. In the MNLS method, all singular values less than $10^{-10}$ times the largest singular value were forced to zero.

Three different source configurations were used and are shown in Figures 1, 2, and 3. The same detector configuration was used for all cases and is shown in Figure 4. Each source/detector configuration was tested for five different values of source and noise variance. Theoretical values of the mean reconstruction error $\eta^{2}$, mean residual $E \chi^{2}$, and mean surprise $\mathrm{E} \rho^{2}$ were computed for each combination of geometry and statistics from the equations given previously. Then ten thousand source distributions were generated, projected, and reconstructed for the same combination. Experimental mean values and 
on Table 1 remain true. The big change is that OCLIM now has a smaller mean error $\eta^{2}$ than any of the other methods. The difference is largest when the noise is small and decreases to insignificance when the noise is large enough.

Computations for configurations 2 and 3 are shown in Tables 3 and 4; the results are similar.

For any of these configurations, the initialization (steps 1.1-1.5) and reconstruction (steps 2.1-2.2) for a hundred data sets take about a second on a workstation class computer. There are only minor differences in the execution time required for the four different reconstruction methods.

The same reconstruction software was used to produce the images shown in figures 5 and 6. Configuration 1 was used with non-uniform priors; specifically, $\alpha_{A}^{2}=1, \alpha_{B}^{2}=0.01$, and $\sigma^{2}=10^{-16}$. A single source dipole was active with amplitude 8 and all other sources were zero. The lefthand plot of each pair was reconstructed with OCLIM; the righthand plot with OWPIM. The dipole is at the position indicated by the dot and is pointing out of the page. Grey shacling indicates areas of current flow into the page.

Figure 5 shows three reconstructions of sources consistent with the priors (that is, sources in region A). The peak produced by OCLIM is consistently higher and narrower than the one produced by OWPIM. For both methods, the peak gets broader and lower as the source gets further from the detector array.

Figure 6 shows two reconstructions of sources inconsistent with the priors. In the first pair, the true source is close to a plausible source and OCLIM maps the true source into a plausible source with substantial artifacts in form of spurious peaks elsewhere. OWPIM finds the true location of the source. In the second pair, the true source is well separated from any plausible source. Both OCLIM and OWPIM find the true source but. OCLIM generates several spurious ridges.

\section{Conclusions}

If the unknown current distribution is expressed as a linear combination of elementary current distributions in fixed positions, then the magnetic field measurements are linear in the unknown source amplitudes. If, in addition, the cost function to be minimized is either the mean square error (reconstructed minus true currents) or the square residual (measured minus reconstructed fields), then the unknown source amplitudes may be found by solving a linear problem. This offers several advantages: The problem is well understood theroretically and software for its solution is readily available. There is only a single, global minimum. Efficient and reliable computer codes for linear algebra are readily available.

If the sources are localized and statistically uncorrelated, then the prior knowledge of the current distribution can be interpreted as a map of expected power dissipation or current density as a function of position. Conversely, such a map derived from geological 


\section{References}

[1] Riitta Hari and Risto J. Ilmoniemi. Cerebral magnetic fields. CRC Crit. Rev. Biomed. Eng., 14(2):93-126, 1986.

[2] Jukka Sarvas. Basic mathematical and electromagnetic concepts of the biomagnetic inverse problem. Phys. Med. Biol., 32(1):11-22, 1987.

[3] Susumu Sato, Marshall Balish, and Robert Muratore. Principles of magnetoencephalography. J. Clin. Neurophysiol., 8(2):144-156, April 1991.

[4] A. van Oosterom. History and evolution of methods for solving the inverse problem. J. Clin. Neurophysiol., 8(4):371-380, October 1991.

[5] Gerhard Stroink. Cardiomagnetic imaging. In Barry L. Zaret, Leon Kaufman, Alan S. Berson, and Rosalie A. Dunn, editors, Frontiers in Cardiovascular Imaging, chapter 11, pages 161-177. Raven Press, New York, 1993.

[6] J. S. Kallman and J. G. Berryman. Weighted least-squares criteria for electrical impedance tomography. IEEE Trans. Med. Imaging, 11(2):284-292, June 1992.

[7] J. G. Berryman and R. V. Kohn. Variational constraints for electrical impedance tomography. Phys. Rev. Lett., 65(3):325-328, 16 July 1990.

[8] B[ernd] Lütkenhöner, K. Lehnertz, M. Hoke, and C. Pantev. On the biomagnetic inverse problem in the case of multiple dipoles. Acta Oto-Laryngologica, Suppl 491:94 $105,1991$.

[9] Manbir Singh, R. Richardo Brechner, and Victor W. Henderson. Neuromagnetic localization using magnetic resonance images. IEEE Trans. Med. Imaging, MI-11(1):129134, March 1992.

[10] M. Singh, D. Doria, V. W. Henderson, G. C. Huth, and J. Beatty. Reconstruction of images from neuro-magnetic fields. IEEE Trans. Nuclear Sci., NS 31(1):585 589, 1984.

[11] Brian Jeffs, Richard Leahy, and Manbir Singh. An evaluation of methods for neuromagnetic image reconstruction. IEEE Trans. Biomed. Eng., BME-34(9):713 723, September 1987.

[12] Jia-Zhu Wang, Samuel J. Williamson, and Lloyd Kaufman. Magnetic source images determined by a lead-field analysis: The unique minimum-norm least-squares estimation. IEEE Trans. Biomed. Eng., BME-39(7):665-675, July 1992.

[13] Jia-Zhu Wang. Minimum-norm least-squares estimation: Magnetic source images for a spherical model head. IEEE Trans. Biomed. Eng., BME-40(4):387-396, April 1993.

[14] Ceon Ramon, Michael G. Meyer, Alan C. Nelson, Francis A. Spelman, and Jeff Lamping. Simulation studies of biomagnetic computed tomography. IEEE Trans. Biomed. Eng., BME-40(4):317-322, April 1993. 
[32] Thomas F. Budinger, Grant T. Gullberg, and Ronald H. Huesman. Emission computed tomography. In Gabor T. Herman, editor, Image Reconstruction from Projections, volume 32 of Topics in Applied Physics, chapter 5, pages 147-246. SpringerVerlag, 1979.

[33] Avinash C. Kak and Malcolnn Slaney. Principles of Computerized Tomographic Imaging. IEEE Press, 1988.

[34] Donald W. Marquardt. An algorithm for least-squares estimation of nonlinear parameters. J. Soc. Indust. Appl. Math., 11(2):431-441, June 1963.

[35] Donald W. Marquardt. Generalized inverses, ridge regression, biased linear estimation, and nonlinear estimation. Technometrics, 12(3):591-612, August 1970.

[36] Andrei Nikolnevich Tikhonov and Vasiliy Y. Arsenin. Solutions of Ill-posed Problem.s. Halsted Press, 1977.

[37] E. Anderson, Z. Bai, C. Bischof, J. Demmel, J. Dongarra, J. Du Croz, A. Greenbaum, S. Hammarling, A. McKenney, S. Ostrouchov, and D. Sorenson. LAPACK Users' Guide. Society for Industrial and Applied Mathematics, Philadelphia, 1992. 
Optimal Constrained Linear Inverse Method

Corollary: Since all covariance matrices are symmetric and positive semidefinite, A. $\boldsymbol{\Sigma}$, and $\mathbf{B}$ are symmetric positive definite and have Cholesky decompositions in the forms

$$
\mathbf{A}=\mathbf{R R}^{T}, \quad \boldsymbol{\Sigma}=\mathbf{S S}^{T}, \quad \text { and } \quad \mathbf{B}=\mathbf{L}^{T}
$$

where $\mathbf{R}, \mathbf{S}$, and $\mathbf{L}$ are all lower triangular and invertible.

Proposition: If $\mathbf{x}$ is linearly independent, then $\mathbf{E}\|\mathbf{D x}\|^{2}>0$ for every matrix $\mathbf{D} \neq 0$.

Proof: Suppose that $E\|\mathbf{D} \mathbf{x}\|^{2}=0$. Then, almost surely, $\|\mathbf{D} \mathbf{x}\|=0$, which implies that $\mathbf{D x}=0$, which implies that $\mathbf{x}$ is linearly dependent. 


\section{Optimal Constrained Linear Inverse Method}

Table 2. 'This table shows how the reconstruction quality varies with noise level when the four different methods are used on configuration 1 with non-uniform priors.

OCLIM
OWPIM
OTPIM MNIS

Configuration 1 with $\sigma^{2}=1.000 \times 10^{-20}, a_{A}^{2}=1.000, a_{B}^{2}=0.010, S N R=245.8$ db, and 100000 data sets:
$\eta^{2} \quad$ theo 4.486
10.727
11.419
$2.417 \times 10^{5}$
expr $\quad 4.456 \pm 0.025$
$10.709 \pm 0.041$
$11.400 \pm 0.043$
$(2.391 \pm 0.021) \times 10^{5}$
$E x^{2}$
101.352
98.999
expr
$101.172 \pm 0.141$
$98.809 \pm 0.140$
100.316
$100.117 \pm 0.143$
84.999
$E \rho^{2}$ theo
42.651
231.248
256.449
$84.797 \pm 0.130$
expr $\quad 42.763 \pm 0.090$
$232.150 \pm 0.821$
$257.459 \pm 0.943$
$8.441 \times 10^{6}$
$(8.395 \pm 0.081) \times 10^{6 i}$

Configuration 1 with $\sigma^{2}=1.000 \times 10^{-16}, \alpha_{A}^{2}=1.000, \alpha_{B}^{2}=0.010,5 N R=165.8 \mathrm{db}$, and 10000 datat sets:
$\eta^{2} \quad$ theo $\quad 10.209$
17.039)
17.519
$2.417 \times 10^{9}$
expr $\quad 10.205 \pm 0.041$
$17.002 \pm 0.054$
$17.492 \pm 0.055$
$(2.391 \pm 0.021) \times 10^{9}$
$E^{2}$ theo
114.253
111.774
111.585
85.000
$\begin{aligned} & E \rho^{2} \text { theo } \\ & \operatorname{expr}\end{aligned}$
$114.161 \pm 0.150$
$111.688 \pm 0.149$
$111.483 \pm 0.150$
$84.797 \pm 0.130$
225.663
257.338
$225.201 \pm 0.775$
$257.600 \pm 0.927$
$8.443 \times 10^{10}$
$(8.395 \pm 0.081) \times 10^{10}$

Configuration I with $\sigma^{2}=1.000 \times 10^{-12}, \alpha_{A}^{2}=1.000, \alpha_{B 3}^{2}=0.010, S N R=85.8 \mathrm{db}$, and 10000 dat.a sets:

$\begin{array}{llllll}\eta^{2} & \text { theo } & 17.366 & 22.307 & 22.939 & 2.417 \times 10^{13} \\ & \text { expr } & 17.403 \pm 0.057 & 22.313 \pm 0.064 & 22.936 \pm 0.065 & (2.391 \pm 0.021) \times 10^{13} \\ E_{\chi^{2}} & \text { theo } & 128.069 & 125.447 & 126.082 & 85.000 \\ & \text { expr } & 127.872 \pm 0.159 & 125.267 \pm 0.158 & 125.880 \pm 0.160 & 84.797 \pm 0.130 \\ E_{\rho^{2}}{ }^{2} & \text { theo } & 15.931 & 169.524 & 211.813 & 8.443 \times 10^{1.4} \\ & \operatorname{expr} & 15.905 \pm 0.053 & 168.929 \pm 0.697 & 211.0^{\prime 3} \pm 0.971 & (8.395 \pm 0.081) \times 10^{14}\end{array}$

Configuration I with $\sigma^{2}=1.000 \times 10^{-6}, \sigma_{A}^{2}=1.000, \alpha_{B}^{2}=0.010, S N R=5.8 \mathrm{db}$, and 10000 dat a sets:
$\eta^{2} \quad$ theo
24.393
20.655
27.093
$2.417 \times 10^{17}$
expr $\quad 24.404 \pm 0.069$
$26.644 \pm 0.072$
$27.089 \pm 0.073$
$(2.391 \pm 0.021) \times 10^{17}$
$\begin{array}{rr}\mathrm{N}^{2} & \text { theo } \\ \text { expr }\end{array}$
139.623
138.394
$E \rho^{2}$
$139.386 \pm 0.167$
$138.158 \pm 0.166$
141.165
74.427
4.377
$4.32 i \pm 0.02 i$
$73.8 \cdot 11 \pm 0.430$
$140.937 \pm 0.170$
85.000
88.174
$84.797 \pm 0.130$
expr
$87.058 \pm 0.022$
$8.443 \times 10^{18}$
$(8.3915 \pm 0.081) \times 10^{1 \times}$

Configuration 1 with $\sigma^{2}=1.000 \times 10^{-4}, \alpha_{A}^{2}=1.000, \alpha_{B}^{2}=0.010, \sin R=-74.2$ db, and 10000 data sets:
$\eta^{2} \quad$ theo
28.333
28.349
28.360
$2.417 \times 10^{21}$
expr $\quad 28.303 \pm 0.075$
$28.320 \pm 0.075$
$28.331 \pm 0.075$
$(2.391 \pm 0.021) \times 10^{21}$
$E x^{2}$
143.973
143.973
144.028
expr
$143.704 \pm 0.170$
$143.705 \pm 0.170$
$143.760 \pm 0.170$
85.000
$E \rho^{2}$ theo
0.027
0.653
0.000
$84.797 \pm 0.130$
expr $\quad 0.027 \pm 0.000$
$0.649 \pm 0.008$
$0.000 \pm 0.000$
$8.443 \times 10^{22}$
$(8.3915 \pm 0.081) \times 10^{22}$ 


\section{Optimal Constrained Linear Inverse Method}

Table 4. This table shows how the rec mstruction quality varies with noise level when the four different methods are used on configuration 3 with uniform priors

OCLIM
OWPIM
OTPIM

MNLS

Configuration 3 with $\sigma^{2}=1.000 \times 10^{-20}, \alpha^{2}=1.000, S N R=251.0 \mathrm{db}$, and 10000 data sets:
$\eta^{2} \quad$ theo
26.490
26.499
28.702
$0.029 \times 10^{5}$
expr $26.471 \pm 0.069$
$26.471 \pm 0.069$
$28.680 \pm 0.074$
$(9.140 \pm 0.071) \times 10^{5}$
$E x^{2}$
42.533
42.483
42.017
expr
$42.414 \pm 0.089$
$42.431 \pm 0.089$
$41.951 \pm 0.093$
20.997
$E \rho^{2} \quad$ theo 101.510
101.491
106.684
$101.284 \pm 0.140$
$106.510 \pm 0.149$
$20.939 \pm 0.065$
$9.014 \times 10^{8}$
$(9.141 \pm 0.071) \times 10^{5}$

Configuration 3 with $\sigma^{2}=1.000 \times 10^{-16}, \alpha^{2}=1.000, S N R=171.0 \mathrm{db}$, and 10000 data sets:
$\eta^{2}$
theo
54.663
54.663
57.426
$9.020 \times 10^{4}$
expr $\quad 54.655 \pm 0.102$
$54.655 \pm 0.102$
$57.386 \pm 0.106$
$(9.140 \pm 0.071) \times 10^{9}$
$E_{1}^{2}$ theo
70.663
70.662
72.235
21.000
$\begin{aligned} & E \rho^{2} \text { theo } \\ & \operatorname{expr}\end{aligned}$
$70.540 \pm 0.116$
$70.540 \pm 0.116$
$72.050 \pm 0.123$
$20.939 \pm 0.065$
73.337
77.426
$0.029 \times 10^{9}$
73.337
$73.100 \pm 0.119$
$77.242 \pm 0.129$
$(9.140 \pm 0.071) \times 10^{9}$

Configuration 3 with $\sigma^{2}=1.000 \times 10^{-12}, \alpha^{2}=1.000, S N R=91.0 \mathrm{db}$, and 10000 dath sets:

$\begin{array}{llllll}\eta^{2} & \text { theo } & 86.192 & 86.192 & 89.808 & 9.029 \times 10^{13} \\ & \text { expr } & 86.165 \pm 0.128 & 86.165 \pm 0.128 & 89.745 \pm 0.132 & (9.140 \pm 0.071) \times 10^{13} \\ E_{\chi^{2}} & \text { theo } & 102.192 & 102.192 & 105.511 & 21.000 \\ & \text { expr } & 102.047 \pm 0.139 & 102.047 \pm 0.139 & 105.360 \pm 0.147 & 20.939 \pm 0.065 \\ E_{\rho^{2}} & \text { theo } & 41.808 & 41.808 & 45.808 & 9.029 \times 10^{13} \\ & \text { expr } & 41.632 \pm 0.087 & 41.632 \pm 0.087 & 45.619 \pm 0.101 & (9.1 .40 \pm 0.071) \times 10^{13}\end{array}$

Configuration 3 with $\sigma^{2}=1.000 \times 10^{-8}, \alpha^{2}=1.000, S N R=11.0 \mathrm{db}$, and 10000 data sets.
$\begin{array}{lll}y^{2} & \text { theo } & 117.197 \\ & \text { expr } & 117.049 \pm 0.151\end{array}$
117.197
119.933
$0.029 \times 10^{17}$
$E_{1}{ }^{2}$ theo
133.197
$117.049 \pm 0.151$
$119.771 \pm 0.154$
$(9.140 \pm 0.071) \times 10^{17}$
expr
$133.137 \pm 0.160$
133.197
$133.137 \pm 0.160$
137.076
21.000
$\begin{aligned} & E \rho^{2} \text { theo } \\ & \text { expr }\end{aligned}$
10.803
10.803
$137.034 \pm 0.167$
$20.939 \pm 0.065$
11.933
(1). 029$) \times 10^{17}$
$10.760 \pm 0.040$
$10.700 \pm 0.040$
$11.859 \pm 0.054$
$(9.1 .10 \pm 0.071) \times 10^{17}$

Configuration 3 with $\sigma^{2}=1.000 \times 10^{-4}, \alpha^{2}=1.000, S N R=-69.0 \mathrm{db}$, and 10000 data sets:

\begin{tabular}{llllll}
$\eta^{2}$ & theo & 127.950 & 127.950 & 128.000 & $9.029 \times 10^{21}$ \\
& expr & $127.710 \pm 0.159$ & $127.710 \pm 0.159$ & $127.760 \pm 0.159$ & $(9.140 \pm 0.071) \times 10^{21}$ \\
$E_{X^{2}}$ & theo & 143.950 & 143.950 & 144.051 & 21.000 \\
& expr & $143.904 \pm 0.168$ & $143.904 \pm 0.168$ & $144.004 \pm 0.168$ & $20.939 \pm 0.065$ \\
$E^{2}$ & theo & 0.050 & 0.050 & 0.000 & $9.029 \times 10^{21}$ \\
& expr & $0.050 \pm 0.000$ & $0.050 \pm 0.000$ & $0.000 \pm 0.000$ & $(9.140 \pm 0.071) \times 10^{21}$ \\
\hline
\end{tabular}



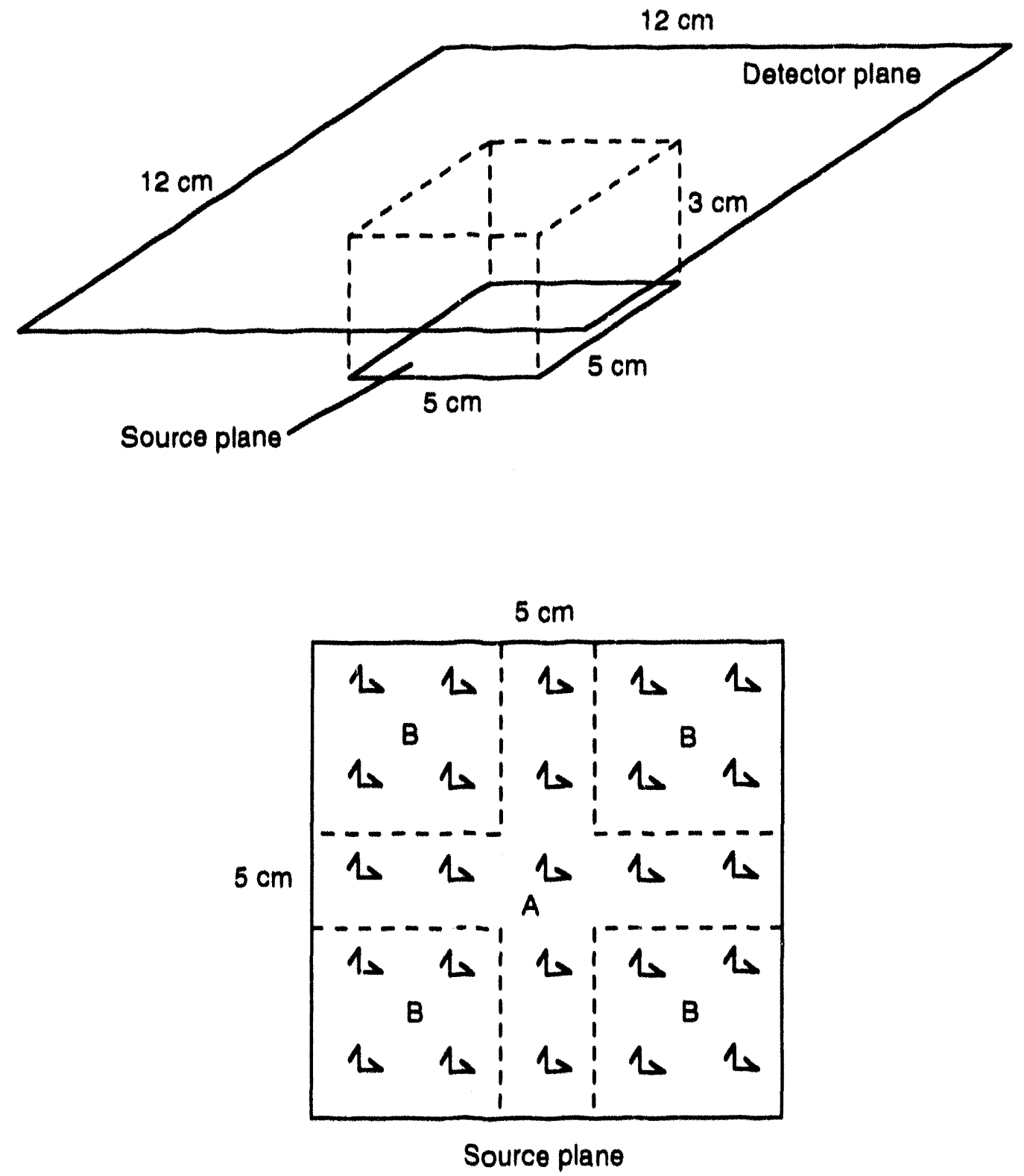

Figure 2. Test configuration 2. The source plane is parallel to the detector plane and contains 25 dipole pairs parallel to the detector plane. Dipoles in region A have higher expected power. 
Optimal Constrained Linear Inverse Method
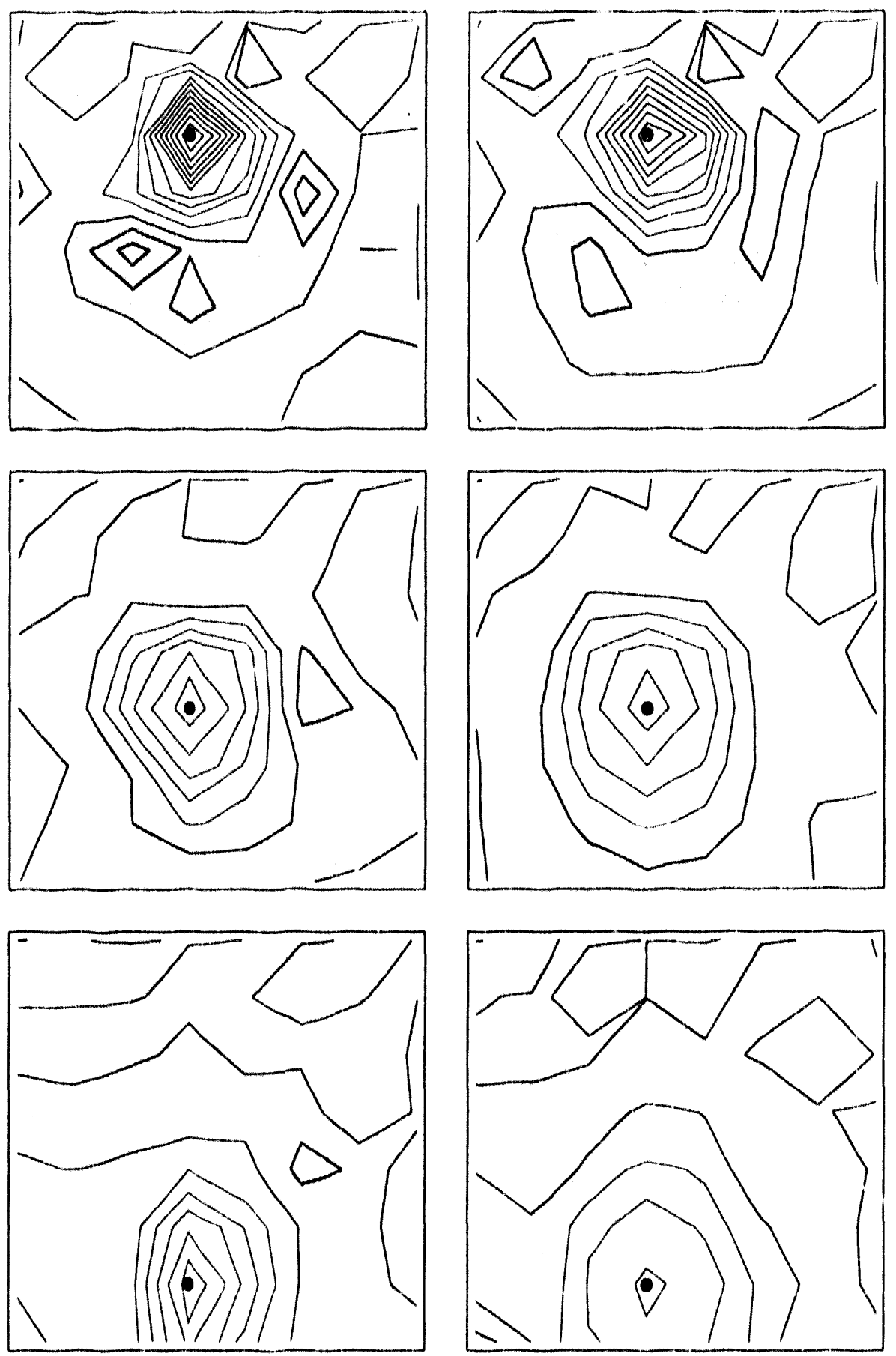

Figure 5. Reconstruction of sources consistent with the prior knowledge. The lefthand image of each pair shows the reconstruction using OCLIM with prior information; the righthand image shows the reconstruction using OWPIM with uniform priors. The true distribution is a single dipole out of the page at the position indicated by the dot. Grey shading indicates areas of current flow into the page. 


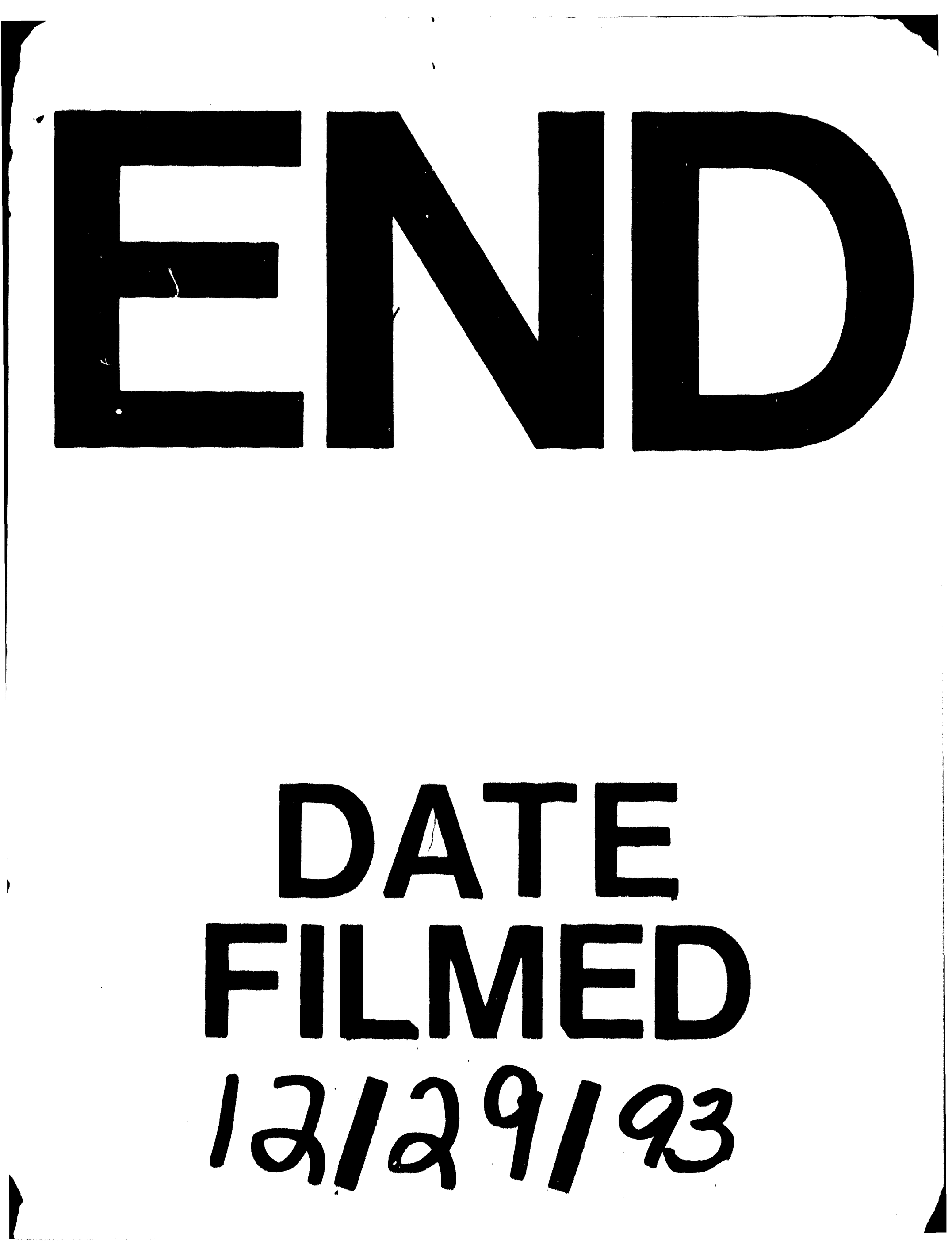


